\title{
Genetic Algorithm for Adaptable Radar Bandwidth
}

\author{
Anthony Martone, Kenneth Ranney, and Kelly Sherbondy \\ U.S. Army Research Laboratory \\ 2800 Powder Mill Road, Adelphi, MD 21042, USA
}

\begin{abstract}
The spectrum sensing, multi-optimization (SS-MO) technique was recently investigated to enhance radar performance when the radar operates in the presence of radio frequency interference (RFI). The SS-MO technique passively monitors the operating band of the radar using spectrum sensing to identify a frequency sub-band with minimal RFI, thus allowing the radar to maintain a high signal to inference plus noise ratio (SINR). Prior results have indicated significant improvement of SINR and PSLR (peak to average sidelobe level ratio) at the cost of a high computational complexity. In this paper, the non-dominated sorting genetic algorithm II (NSGA-II) is used to lower the computational complexity while maintaining performance.
\end{abstract}

Keywords-radar, spectrum sharing, spectrum sensing, multiobjective optimization, Pareto-optimal solutions, waveform agility

\section{INTRODUCTION}

In January 2015 the Federal Communications Commission (FCC) raised over $\$ 41$ billion dollars in the auction of Advanced Wireless Service (AWS) licenses, approximately four times more revenue than originally expected [1]. These licenses grant commercial wireless devices access to three auctioned bands, the AWS-03 bands, located at 1695-1710 $\mathrm{MHz}, 1755-1780 \mathrm{MHz}$, and $2155-2180 \mathrm{MHz}$. This auction is just the beginning. Other frequency bands are being considered for auction, including the $5 \mathrm{GHz}$ band $(5.15-5.925 \mathrm{GHz})$, and will have a tremendous impact on government radar systems [2]. Government radar systems that currently operate in an auctioned band are given the choice to relocate or share the band with the commercial wireless devices. Military Program Executive Officers (PEOs) and Program Managers (PMs) will be faced with the following Program of Record (POR) challenges for their core radar systems [3]: 1) mitigating radio frequency interference (RFI) generated from commercial wireless devices in order to maximize radar performance; 2) avoid causing RFI to the commercial wireless devices; 3) share the spectrum with commercial wireless devices.

Solutions for mitigating RFI in radar include [4]: 1) random frequency selection; 2) frequency notching methods; 3) subspace techniques; and 4) RFI reconstruction and subtraction. As discussed in [4], the effectiveness of these solutions requires an estimate of the RFI parameters, a challenging task. Furthermore, these solutions do not address radars capability to avoid causing RFI in the commercial wireless devices. Solutions for joint radar and communication system spectrum sharing require cooperative policies that utilize time, frequency, phase, and/or space domain strategies [5-7]. The challenge for many spectrum sharing approaches is the ambient RFI generated by neighboring RF systems operating in the vicinity of the joint radar and communication system; coexistence between the joint radar and communication system and the neighboring system is therefore not possible.
A potential spectrum sharing solution that addresses the above radar challenges is the spectrum sensing, multi-objective optimization (SS-MO) technique originally introduced by the authors in [8]. This technique passively monitors the operating band of the radar using spectrum sensing to identify a frequency sub-band with minimal RFI. The proposed technique uses the weighted sum multi-objective optimization algorithm (WSMO) to maximize the signal to interference plus noise ratio (SINR) and bandwidth. It has been shown that this technique significantly improves SINR and the peak-to-average sidelobe ratio [9].

The computational complexity of WSMO is of order $O\left(N^{2}\right)$, where $N$ is the number of processed samples. Implementation of WSMO is therefore inadequate for radar applications that require fast solutions for target detection and classification. The computational complexity of WSMO also results in an inaccurate estimate of fast-changing RFI, where the RFI changes at a rate faster than SS-MO convergence. The computational complexity of the SS-MO technique must therefore be reduced.

WSMO combines two objective functions into a single composite function [10]. The composite function is maximized to determine a single solution. Other multi-objective optimization techniques search for the Pareto optimal set, a surface of non-dominated solutions. This set is optimal in the sense that no one solution is better or worse than another. The Pareto optimal set is large for many problems and multiobjective optimization is reduced to finding the best-known Pareto optimal set for given computational requirements. The solutions in the best-known Pareto optimal set must maintain diversity in order to represent all solutions equally (including the extreme ends).

Genetic algorithms are well suited at solving multi-objective optimization problems since they search different regions of the solution space in parallel, allowing for complex solutions with non-convex, discontinuous, and multimodal solution spaces [10]. The genetic algorithm considered here is the nondominated sorting genetic algorithm II (NSGA-II), a wellknown and widely accepted genetic algorithm. NSGA-II [11]: 1) uses a non-dominating sorting approach of low computational complexity with an elite population to significantly speed-up performance; 2 ) preserves diversity to maintain a good spread of solutions.

In this paper NSGA-II is compared to WSMO. Section II of this paper outlines the proposed SS-MO technique and formulates both WSMO and NSGA-II approaches. Section III describes a spectrum sensing experiment to collect multiple frequency spectra of different spectral occupancy, and examines NSGA-II for multiple input parameters. The 
frequency spectra are then analyzed in Section IV using both approaches. Finally, conclusions are drawn in Section V.

\section{SPECTRUM SENSING FOR RADAR}

An overview of the proposed SS-MO technique is discussed in this section. Sections II-A and II-B describe the implementation of the SS-MO technique using WSMO and NSGA-II (respectively). A block diagram of the SS-MO technique for radar is illustrated in Fig. 1. Spectrum sensing is first implemented to passively monitor the operating band, $B$, of the radar while the radar remains inactive; $B$ is the full bandwidth. After the spectrum sensing process is complete, the radar begins operation using the full bandwidth $B$ until the SSMO technique converges to a solution. The Radar System then synthesizes the appropriate waveform using the optimal frequency and bandwidth information provided by SS-MO. The synthesized waveform is then transmitted at the start of the next coherent processing interval (CPI).

The spectrum sensing receiver receives, processes, and digitizes the observed RF spectrum to generate a sequence of $N$ time domain samples. These samples are converted to the power spectrum $\Theta=\left\{\theta_{1}, \ldots \theta_{N}\right\}$, via the fast Fourier transform (FFT), for the frequencies $F=\left\{f_{1}, \ldots f_{N}\right\}$ with frequency resolution $F_{r}$. The spectrum occupancy for $\Theta$ is defined as the percentage of RFI over the threshold $\Omega$ :

$$
P O=\frac{100}{N} \sum_{n=1}^{N} y_{n},
$$

where $y_{n}$ is defined using the following binary hypothesis test:

$$
y_{n}=\left\{\begin{array}{ll}
1, & \theta_{n} \geq \Omega \\
0, & \theta_{n}<\Omega
\end{array} .\right.
$$

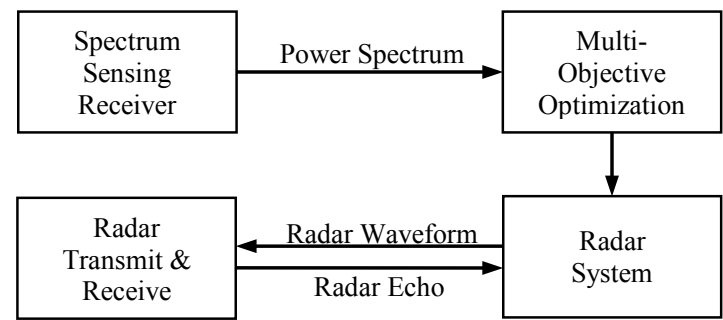

Fig. 1. Spectrum sensing, multi-optimization technique for radar.

The power spectrum is used to form the SINR objective function:

$$
Z_{l}\left(\beta_{i}, f_{j}\right)=P_{r}\left(\beta_{i}\right) / \Gamma\left(\beta_{i}, f_{j}\right),
$$

where $P_{r}\left(\beta_{i}\right)$ is the receive power modeled using the radar range equation. $\Gamma\left(\beta_{i}, f_{j}\right)$ is an empirical estimate of the interference and noise for the $i^{\text {th }}$ sub-band, $\beta_{i}$. The $i^{\text {th }}$ sub-band is defined as $\beta_{i}=$ $i F_{r}$ for $i=\{1, \ldots N\}$. The $j^{t h}$ start frequency of a given sub-band is defined as $f_{j} \in F$ for $j=\{1, \ldots N\}$. The empirical estimate of $\Gamma\left(\beta_{i}, f_{j}\right)$ is formed deterministically using WSMO (Section II-A) and randomly using NSGA-II (Section II-B). The first goal of the SS-MO technique is to adjust $\beta_{i}$ and $f_{j}$ in order to maximize (2). The receive power is defined as

$$
P_{r}\left(\beta_{i}\right)=P_{t} G^{2} \lambda^{2} \sigma \tau N_{P} \beta_{i} /\left[(4 \pi)^{3} R^{4}\right]=C \beta_{i},
$$

where $P_{t}$ is the peak transmit power of the radar, $G$ is the antenna gain, $\lambda$ is the wavelength of the carrier frequency, $R$ is the range to target, $\tau$ is the radar pulse width, $N_{P}$ is the number of pulses transmitted by the radar within the CPI, and $C$ is a constant that replaces the product of the constant radar parameters (for simplicity). Eq. (3) supports a pulse compression waveform with the time-bandwidth (TB) product $\tau \beta_{i}$, however the SS-MO technique is modifiable to support multiple waveform types. The sub-band size is the second objective function and defined as

$$
Z_{2}(\beta)=\beta_{i} \text {. }
$$

The second goal of the SS-MO technique is to adjust $\beta_{i}$ in order to maximize (4); this occurs when $\beta_{i}=B$.

Notice that the presence of $\beta_{i}$ in both (2) and (4) sets up a fundamental conflict. In order to maximize (4), we require $\beta_{i}$ to be as large as possible. This implies, however, that more RFI is present as $\beta_{i}$ increases thereby decreasing SINR. Multi-objective optimization is used to maximize these conflicting objective functions by defining the decision variable $x=\left\{\beta_{i}, f_{j}\right\}$ that forms the solution space $X$. The goal of the optimization process is to find the optimal solution, $x^{*}=\left\{\beta_{i}^{*}, f_{j}^{*}\right\}$, such that the objective functions in (2) and (4) are maximized:

$$
Z\left(\beta_{i}^{*}, f_{j}^{*}\right)=\left\{Z_{1}\left(\beta_{i}^{*}, f_{j}^{*}\right), Z_{2}\left(\beta_{i}^{*}\right)\right\}
$$

subject to $Z_{1}\left(\beta_{i}{ }^{*}, f_{j}^{*}\right) \geq Z_{1, \min }$ and $Z_{2}\left(\beta_{i}{ }^{*}\right) \geq Z_{2, \min }$, where $Z_{1, \min }$ and $Z_{2, \min }$ are the boundary conditions for minimum SINR and bandwidth (respectively) allowable for radar operation. The solution in (5) is considered feasible if it satisfies these boundary conditions. For a given power spectrum $\theta_{n}$, the maximum value of SINR is found as

$$
Z_{1, \max }=\max \left[Z_{1}\left(\beta_{i}, f_{j}\right)\right] \text {. }
$$

\section{A. Weighted Sum Multi-Objective Optimization}

WSMO uses the following recursive algorithm (exhaustive search) to form the empirical estimate of interference and noise:

$$
\Gamma\left(\beta_{i}, f_{j}\right)=\left\{\begin{array}{cc}
\theta_{j}, & i=1, j=1, \ldots N \\
\frac{\Gamma\left(\beta_{1}, f_{j}\right)+\Gamma\left(\beta_{1}, f_{j+1}\right)}{2}, & i=2, j=1, \ldots N-1 \\
\frac{\Gamma\left(\beta_{i-1}, f_{j}\right)+\Gamma\left(\beta_{1}, f_{i+j-1}\right)}{i} & i=3, \ldots N, j=1, \ldots N-i+1
\end{array} .\right.
$$

The following linear weighting function is next used to combine the objective functions defined in (2) and (4):

$$
Z\left(\beta_{i}, f_{j}\right)=\alpha \dot{Z}_{1}\left(\beta_{i}, f_{j}\right)+(1-\alpha) \dot{Z}_{2}\left(\beta_{i}\right)
$$

where $0 \leq \alpha \leq 1$ is the weighting parameter, $\dot{Z}_{1}\left(\beta_{i}, f_{j}\right)$ is the normalized objective function of $Z_{l}\left(\beta_{i}, f_{j}\right)$, and $\dot{Z}_{2}\left(\beta_{i}\right)$ is the normalized objective function of $Z_{2}\left(\beta_{i}\right)$. Then the optimal subband and frequency pair, $\left(\beta_{i}^{*}, f_{j}^{*}\right)$, is solved using:

$$
\left(\beta_{i}^{*}, f_{j}^{*}\right)=\underset{\left(\beta_{i}, f_{j}\right)}{\arg \max }\left[Z\left(\beta_{i}, f_{j}\right)\right] .
$$

The computational complexity of the weighted average approach, as reported in [8], results in $N^{2}$ summations and $\left(3 N^{2}+2 N\right)$ multiplications/divisions, and is of order $O\left(N^{2}\right)$.

\section{B. Genetic Algorithm for Multi-Objective Optimization}

An overview of NSGA-II for the SS-MO technique is discussed in this section; a full description of NSGA-II is included in [11]. A block diagram of NSGA-II for multiobjective optimization is shown in Fig. 2. The genetic algorithm forms a population of $M$ individuals over $T$ generations. A random set of individuals is created for the first generation. The 
set of $M$ individuals for generation $t \in\{1, \ldots T\}$ is defined as $X_{t}$ $=\left\{X_{t, l}, \ldots X_{t, M}\right\}$. Each individual is defined as a candidate decision variable $X_{t, m}=\left\{\beta_{i}, f_{j}\right\}_{t, m}$, where $X_{t, m} \in X_{t}$. The goal of the genetic algorithm is to evolve this population over multiple generations such that the candidate decision variables converge to $\left\{\beta_{i}{ }^{*}, f_{j}^{*}\right\}$. For each generation the quality, or fitness, of the individuals are measured using the SINR and bandwidth objective functions. The objective functions for individual $X_{t, m}$ are defined as

$$
\begin{aligned}
& Z_{l}\left(X_{t, m}\right)=Z_{l}\left(\left\{\beta_{i}, f_{j}\right\}_{t, m}\right)=i C \beta_{i} / \sum_{k=j}^{j+i-1} \theta_{k}, \\
& Z_{2}\left(X_{t, m}\right)=Z_{2}\left(\left\{\beta_{i}\right\}_{t, m}\right)=\beta_{i} .
\end{aligned}
$$

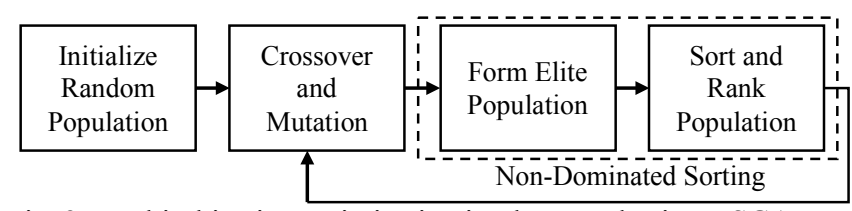

Fig. 2. Multi-objective optimization implemented using NSGA-II.

Crossover and mutation procedures map, or encode, the parents in generation $t, X_{t, m}$, to the children in generation $t+1$, $X_{t+1, m}$. These procedures are done independently for each decision variable, i.e. $\left\{\beta_{i}\right\}_{t, m}$ maps to $\left\{\beta_{i}\right\}_{t+1, m}$ and $\left\{f_{j}\right\}_{t, m}$ maps to $\left\{f_{j}\right\}_{t+1, m}$. Simulated binary crossover (SBX) is used for the crossover procedure [12]. SBX operates directly on the numerical value of $X_{t, m}$ and not the binary representation (hence the name). The input parameter to SBX is $\eta_{c}$, a variable that controls the similarity of the individuals between the parent and child. A large $\eta_{c}$ produces children very similar to the parents, where a small $\eta_{c}$ produces dissimilar children. A small percentage of children are next selected for mutation, where the probability that a child is selected for mutation is defined as $P_{m}$. Polynomial mutation is implemented for the selected individuals. The input parameter to polynomial mutation is $\eta_{m}$, a variable that controls the similarity between the original and mutated individual (with properties similar to $\eta_{c}$ ).

The computational complexity to execute the above crossover and mutation procedures for $M$ individuals over $T$ generations is of order $O(T M N)$. The bottleneck for computational complexity lies with forming the objective function in (10), which requires $i-1$ summations (note that $i$ could equal $N$ ). The number of multiplications/divisions for the crossover and mutation procedures is of order $O(T M)$.

The child and parent populations are next merged to form an elite population $P$ (i.e. generation's $t$ and $t+1$ ):

$$
P=\left\{P_{1}, \ldots P_{2 M}\right\}=\left\{X_{t, 1}, \ldots X_{t, M}, X_{t+1,1}, \ldots X_{t+1, M}\right\}=\left\{X_{t}, X_{t+1}\right\} .
$$

This elite population prevents loss of good solutions from the parent population and has been shown to speed-up performance [11]. A sorting and ranking procedure is next used to separate and sort the feasible and non-feasible individuals in $P$. The set of non-feasible solutions do not satisfy the boundary conditions of (5). Non-feasible solutions are sorted based on their absolute distance from the boundary conditions. Define the sorted set of $\bar{M} \leq M$ non-feasible solutions as $\bar{P}=\left\{\bar{P}_{1}, \ldots \bar{P}_{\bar{M}}\right\} \subset P$. Note that $\bar{P}_{1}$ has the highest (or best) rank and corresponds to the individual closest to the boundary conditions. The computational complexity needed to sort the non-feasible solutions is $O(2 \bar{M})+O(\bar{M} \log \bar{M})$.
The set of feasible solutions satisfy the boundary conditions of (5). The feasible solutions are sorted based on the fast, nondominated sorting procedure. Feasible solutions are first ranked based on a measure of dominance. Dominance between any two individuals $P_{m} \in P$ and $P_{q} \in P$ is defined as follows: $P_{m}$ dominates $P_{q}$ if 1) $Z_{l}\left(P_{m}\right)>Z_{l}\left(P_{q}\right)$ and 2) $Z_{2}\left(P_{m}\right)>Z_{2}\left(P_{q}\right)$; $P_{q}$ dominates $P_{m}$ if 1) $Z_{1}\left(P_{q}\right)>Z_{1}\left(P_{m}\right)$ and 2) $Z_{2}\left(P_{q}\right)>Z_{2}\left(P_{m}\right)$; otherwise $P_{m}$ and $P_{q}$ are non-dominant. Ranks range from $\{1 \ldots$ $K$ \}, where Rank 1 individuals dominate all other individuals. It is possible to have multiple, non-dominating individuals in the same rank. The crowding distance is then used to further discriminate these non-dominating individuals. The crowding distance preserves diversity by favoring isolated individuals (with larger distances); isolated individuals provide uniform, spread-out Pareto optimal solutions. Define the sorted set of $\widetilde{M} \leq M$ feasible solutions as $\widetilde{P}=\left\{\widetilde{P}_{1}, \ldots \widetilde{P}_{\widetilde{M}}\right\} \subset P$. Note that $\widetilde{M}$ $+\bar{M}=M . \widetilde{P}_{l}$ has the highest (or best) rank and is the most dominant individual in the feasible set. The computational complexity needed to sort the feasible solutions is $O(2 \widetilde{M})+$ $O(2 \widetilde{M} \log 2 \widetilde{M})+O(\widetilde{M} \log \widetilde{M})$. The sorted feasible and nonfeasible solution sets are then combined as $\{\widetilde{P}, \bar{P}\}$, where the individuals in $\widetilde{P}$ are ranked higher than those in $\bar{P}$. This process then iterates for $T$ generations.

Execution of NSGA-II (Fig. 2) for a given spectrum in $\Theta$ requires multiple random trials. Random trials are necessary since NSGA-II randomly generates its initial population (random initial conditions) resulting in different solutions per trial. The performance statistics analyzed in this paper considers an average of these random trials.

\section{DATA COLLECTION AND NSGA-II ANALYSIS}

Multiple RF spectra are measured and used for training and experimentation purposes. As will be discussed in this section, the training set is used to identify an appropriate set of input parameters for NSGA-II. The experimentation data is then processed by WSMO and NSGA-II and the results are discussed in Section IV. In order to examine the capabilities of WSMO and NSGA-II, the set of measured data must contain RF spectra with different levels of interference. RF spectra is therefore collected between $100 \mathrm{MHz}$ and $1 \mathrm{GHz}$, a band consisting of various RF interference conditions.

The spectral data is collected using the Agilent Technologies N9342CN handheld spectrum analyzer with an AOR DA3200 ultra-wideband discone antenna. The parameters of the spectrum analyzer are set as follows: $B=100 \mathrm{MHz}$ total bandwidth, $F_{r}=$ $100 \mathrm{kHz},-30 \mathrm{dBm}$ reference level, $20 \mathrm{~dB}$ attenuation value, and no averaging. The thermal noise of the spectrum analyzer is $P_{N}$ $=-92 \mathrm{dBm}$. The spectrum analyzer collects 8000 power spectra by tuning from $100 \mathrm{MHz}$ to $900 \mathrm{MHz}$ in $100 \mathrm{kHz}$ steps; $N=1000$ samples are collected per spectrum. Define the measured power spectra as $\Psi=\left\{\Psi_{1}, \ldots \Psi_{8000}\right\}$, where each $\Psi_{d}$ contains the power samples $\left\{\theta_{1}, \ldots \theta_{1000}\right\}$ and $d \epsilon\{1, \ldots 8000\}$.

The training and experimentation sets are measured using the same spectrum analyzer parameters discussed above, but collected at difference times of the day. The percent occupancy for both the training and experiment sets is shown in Fig. 3. The threshold for PO in (1) is $\Omega=-88 \mathrm{dBm}$ (i.e. the threshold is used to find RFI $3 \mathrm{~dB}$ higher than thermal noise). As shown, the training and experimental sets are similar. 


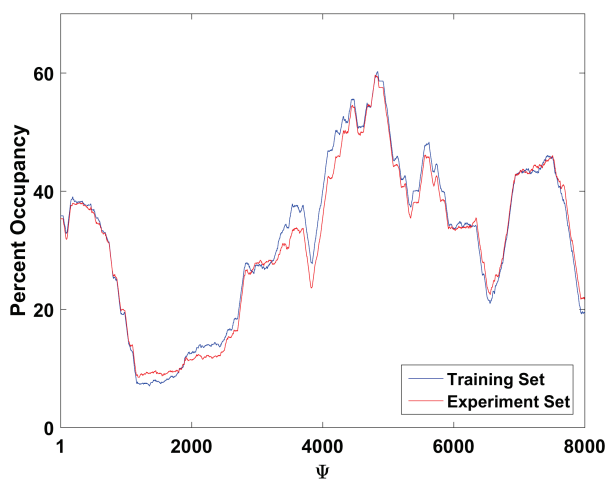

Fig. 3. Spectral occupancy of the measured data. The training and experiment sets were measured at different times during the day.

The training set is used to examine the performance of NSGA-II for multiple input parameters. In order to examine the trade-off between SINR and bandwidth, the performance is measured using the linear, or $L_{l}$, distance metric, which quantifies the error between $\left\{Z_{1}\left(\beta_{i}^{*}, f_{j}^{*}\right), Z_{2}\left(\beta_{i}^{*}\right)\right\}$ and $\left\{Z_{1, \max }, Z_{2, \max }=B\right\}$. Recall that $Z_{1, \max }$ is found using (6). The $L_{1}$ distance for the spectrum $\Psi_{d}$ is defined as:

$$
D_{d}=\frac{Z_{1, \max }-Z_{1}\left(\beta_{i}^{*}, f_{j}^{*}\right)}{Z_{1, \max }}+\frac{B-Z_{2}\left(\beta_{i}^{*}\right)}{B}
$$

NSGA-II is used to process each spectrum in $\Psi$ to generate the set of $L_{l}$ distances $\left\{D_{1}, \ldots D_{8000}\right\}$. Recall that NSGA-II requires multiple random trials, $R$, to generate its initial population; $R=50$ random trials are implemented in this evaluation. The resulting $R$ distance measures for spectrum $\Psi_{d}$ are defined as $\left\{D_{d, 1}, \ldots D_{d, 50}\right\}$. These distance measures are next averaged using $\widehat{D}_{d}=\sum_{k=1}^{R} D_{d, k} / R$ for $\Psi_{d}$. The set of averaged distance measures, $\widehat{D}=\left\{\widehat{D}_{l}, \ldots \widehat{D}_{8000}\right\}$, is then formed for all 8000 spectra.

NSGA-II is evaluated using the following sets of parameters: $\eta_{c} \in\{2.0,2.5,3.0,3.5,4.0\} ; \eta_{p} \in\{2.0,2.5,3.0,3.5,4.0\} ; M \epsilon$ $\{10,20,30,40\} ; T \in\{20,30,40,50\}$. The goal of this examination is to find the parameter set $\left\{\eta_{c}{ }^{*}, \eta_{p}{ }^{*}, M^{*}, T^{*}\right\}$ that results in a high performance implementation of NSGA-II. NSGA-II also uses the following parameters: $Z_{1, \min }=0.1$ ($10 \mathrm{~dB}$ ) for SINR; $Z_{2, \min }=5 \mathrm{MHz}$ for bandwidth; $C=2 \times 10^{-19}$ as the radar range equation constant; $P_{m}=0.1$ as the mutation probability. It should be noted that these parameters are selected to test the capabilities of WSMO and NSGA-II for an arbitrary radar type and application.

NSGA-II is first evaluated to find $\eta_{c}{ }^{*}$ and $\eta_{p}{ }^{*}$ using $T=50$ generations; $T$ is chosen to ensure that the population converges to feasible solutions (which is verified during analysis). This evaluation process must be examined for each population size. For a given $M$, NSGA-II first processes spectrum $\Psi_{d}$ for each combination of $\eta_{c}$ and $\eta_{p}$, resulting in 25 executions of NSGAII. Eq. (12) then produces the set $\left\{\widehat{D}_{d, 1}, \ldots \widehat{D}_{d, 25}\right\}$ of averaged, $L_{1}$ distance measures. The parameter combination corresponding to the smallest error is then defined as $\left\{\tilde{\eta}_{c}, \tilde{\eta}_{m}\right\}=$ $\min _{\eta_{c} \eta_{m}}\left\{\widehat{D}_{d, 1}, \ldots \widehat{D}_{d, 25}\right\}$. Note it is possible that multiple parameter combinations produce the same result, meaning that multiple $\left\{\tilde{\eta}_{c}, \tilde{\eta}_{m}\right\}$ solutions are possible for a given spectrum. This process is repeated for each spectrum and $M$ resulting in 54,474 pairs of $\left\{\tilde{\eta}_{c}, \tilde{\eta}_{m}\right\}$. A histogram is used to evaluate these pairs as shown in Fig. 4. Examination of the histogram indicates that $\eta_{c}{ }^{*}$ $=2.5$ and $\eta_{p}{ }^{*}=3.5$.

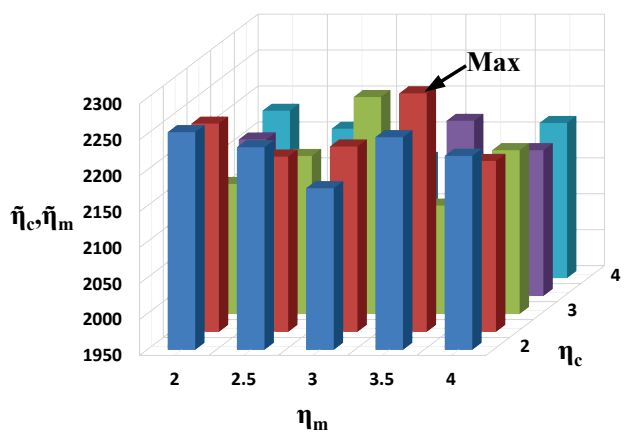

Fig. 4. Histogram used to evaluate the NSGA-II parameters $\eta_{c}$ and $\eta_{p}$.

NSGA-II is next evaluated for $M$ using $\eta_{c}{ }^{*}, \eta_{p}{ }^{*}$, and $T=50$. A set of $L_{1}$ distance measures $\widehat{D}$ (as defined above) is again generated for each population size. Define these measures as $\left\{\widehat{D}_{d, 10}, \widehat{D}_{d, 20}, \widehat{D}_{d, 30}, \widehat{D}_{d, 40}\right\}$, where $\widehat{D}_{d, M}$ denotes population size $M$ for spectrum $\Psi_{d}$. The population size is evaluated using the average estimate $\widetilde{D}_{M}=(1 / 8000) \sum_{k=1}^{8000} \widehat{D}_{k, M}$. The population size is also evaluated using the number of summations since it largely contributes to the computational complexity. The estimate for the summations is formed using the same method as for $\widetilde{D}_{M} . \widetilde{D}_{M}$ and summation results are shown in Fig. 5 for $M$

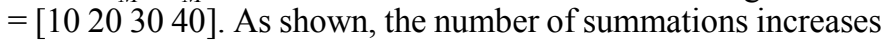
linearly as the population increases. $\widetilde{D}_{M}$ diminishes sharply as the population increases from 10 to 20 , an error difference of 0.035; then more slowly from 20 to 40 , an error difference of 0.013 . To minimize the error, the population size should be 40 ; however, since a small error difference exists between 20 and 40,20 is also a good candidate. Finally, since a population of 20 also reduces computational complexity, the population choice is $M^{*}=20$.

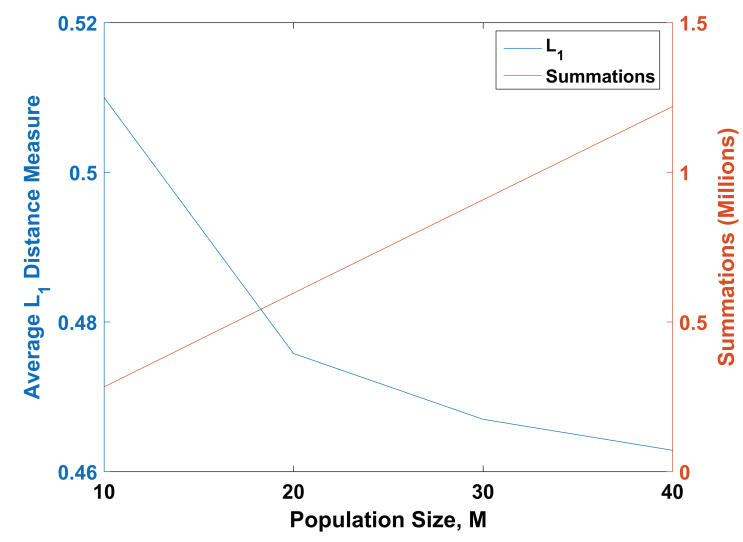

Fig. 5. Analysis of population size for NSGA-II.

NSGA-II is next evaluated for the generation size $T$ with $\eta_{c}{ }^{*}$, $\eta_{p}{ }^{*}$, and $M^{*}$. A set of $L_{l}$ distance measures is generated for each generation size using the same method as the population size. Define these measures as $\left\{\widehat{D}_{d, 20}, \widehat{D}_{d, 30}, \widehat{D}_{d, 40}, \widehat{D}_{d, 50}\right\}$, where $\widehat{D}_{d, T}$ denotes generation size $T$ for spectrum $\Psi_{d}$. The distance measures and summation results are shown in Fig. 6. $\widetilde{D}_{T}$ diminishes sharply as the generation size increases from 20 to 30 , an error difference of 0.025 ; then more slowly from 30 to 50 , 
an error difference of 0.02 . To minimize the error, the generation size should be 50 ; however, since a small error difference exists between 30 and 50, 30 is also a good candidate. Finally, since a population size of 30 also reduces the summation, the generation size is selected to be $T^{*}=30$.

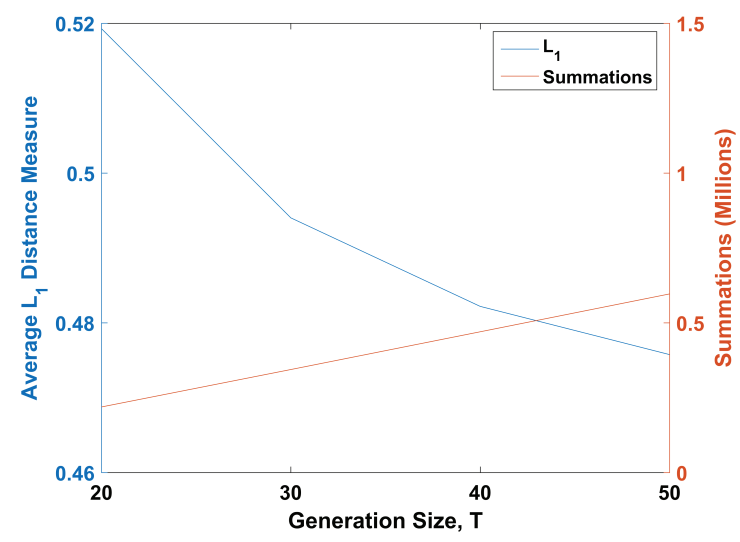

Fig. 6. Analysis of generation size for NSGA-II.

\section{EXPERIMENT AND ANALYSIS}

WSMO and NSGA-II process the experiment data using the parameters specified in Section III. A summary of these parameters are highlighted in Table I. The weighting parameter for WSMO, $\alpha=0.5$, is selected so that each objective function is given equal priority. Performance is measured and compared for: 1) full bandwidth (FB) $B$, representing standard radar operation with no spectrum sensing; 2) WSMO; 3 ) and NSGAII. The performance is measured using SINR, computational complexity, and the $L_{1}$ distance metric. The performance measures used for NSGA-II of the $R$ random trials, where each random trial corresponds to a different initial population. NSGA-II produces $M$ optimized solutions whereas WSMO only produces 1 solution. In order to compare WSMO to NSGA-II, the individual with the lowest $L_{l}$ distance is used to represent NSGA-II.

Table I: Experiment parameters and objective function values.

\begin{tabular}{|c|c|c|c|c|c|c|c|c|c|}
\hline $\boldsymbol{Z}_{1, \boldsymbol{m i n}}$ & $\boldsymbol{Z}_{2, \boldsymbol{m i n}}$ & $\mathbf{C}$ & $\boldsymbol{\alpha}$ & $\boldsymbol{\eta}_{\boldsymbol{c}}{ }^{*}$ & $\boldsymbol{\eta}_{\boldsymbol{m}}{ }^{*}$ & $\boldsymbol{M}^{*}$ & $\boldsymbol{T}^{*}$ & $\boldsymbol{P}_{\boldsymbol{m}}$ & $\boldsymbol{R}$ \\
\hline 0.1 & $5 \mathrm{MHz}$ & $2 x 10^{-19}$ & 0.5 & 2.5 & 3.5 & 20 & 30 & 0.1 & 50 \\
\hline
\end{tabular}

The SINR of the processed spectra is shown in Fig. 7. Six lines are illustrated: 1) the red line for FB; 2 ) the blue line for WSMO; 3) the black line for NSGA-II; 4) the dashed red line for the FB average SINR $(5.23 \mathrm{~dB}) ; 5)$ the dashed blue line for the WSMO average SINR $(10.91 \mathrm{~dB}) ; 6)$ the dashed black line for the NSGA-II average SINR $(10.75 \mathrm{~dB})$. The FB method produces some results below $Z_{1, \text { min }}$ indicating non-feasible solutions. WSMO and NSGA-II met the SINR boundary limit requirements for all processed spectra. Furthermore, these optimization approaches indicate a significant increase in SINR as compared with the FB approach. Although the average SINR result for WSMO is slightly higher than that of NSGA-II, the difference is less than $0.2 \mathrm{~dB}$.

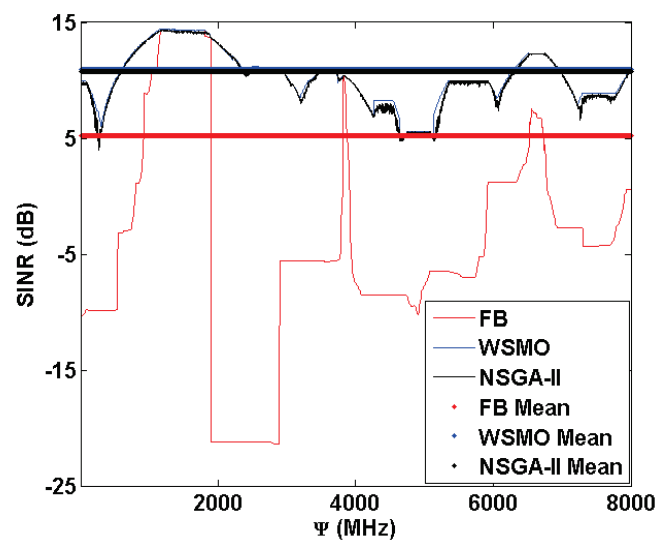

Fig. 7. SINR results for FB, WSMO, and NSGA-II. WSMO and NSGA-II indicate a significant increase in SINR compared to FB.

The $L_{1}$ distance measure is shown in Fig. 8 where WSMO serves as the lower bound. The WSMO mean, 0.43 , and the NSGA-II mean, 0.48, are significantly less than the FB mean, 0.85 . This result further justifies the use of the SS-MO technique compared to the FB approach. Fig. 8 also illustrates that NSGAII results in the highest $L_{l}$ distance measures (as compared to FB) for a small number of spectra. These spectra are located between $\Psi_{203}$ and $\Psi_{293}$ (corresponding to $120.3-229.3 \mathrm{MHz}$ ) and $\Psi_{1152}$ and $\Psi_{1821}$ (corresponding to $215.2-382.1 \mathrm{MHz}$ ). The spectra between $\Psi_{1152}$ and $\Psi_{1821}$ represent thermal noise (i.e. the noise floor) without the presence of RFI. The best solution for thermal noise is to select the full bandwidth. Examination of the NSGA-II results indicate that the full bandwidth is not consistently selected, however NSGA-II does generate solutions "close to" the full bandwidth.

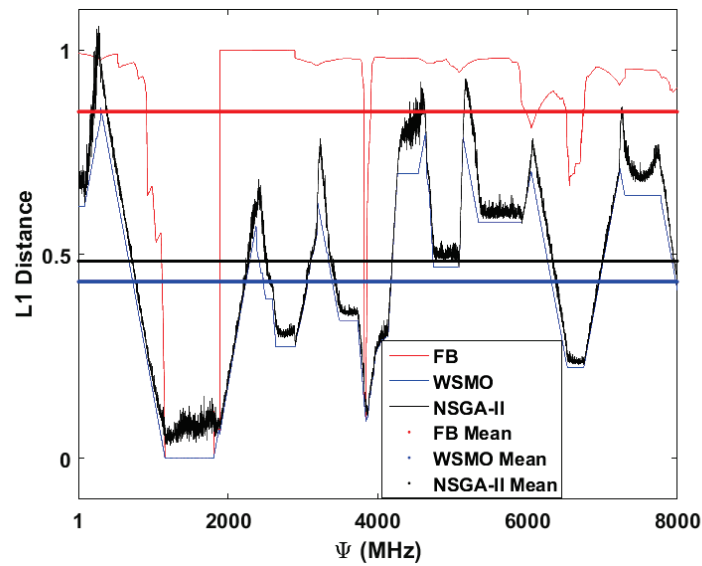

Fig. 8. $L_{1}$ distance measure results for FB, WSMO, and NSGA-II.

The spectra between $\Psi_{203}$ and $\Psi_{293}$ contain multiple RFI subbands; an example spectrum $\left(\Psi_{274}\right)$ is shown in Fig. 9a. When processing these spectra, NSGA-II generates very different results for each of the $R$ random trials. Fig. 9b illustrates the variance of the start and end frequencies of the $R$ random trials for each spectrum. A large variation is indicated for the spectra between $\Psi_{203}$ and $\Psi_{293}$. Other large variance locations (i.e. $\Psi_{2390}$, $\left.\Psi_{3221}, \Psi_{4629}, \Psi_{5172}, \Psi_{6056}, \Psi_{7249}, \Psi_{7774}\right)$ also correspond to spectra with multiple sub-bands of RFI. Hence, when processing RF spectra containing multiple sub-bands of RFI: 1) NSGA-II does not consistently converge to the best-known Pareto optimal set; 
2) selection of $\left\{\beta_{i}^{*}, f_{j}^{*}\right\}$ by NSGA-II is highly dependent on the random locations of the initial population.

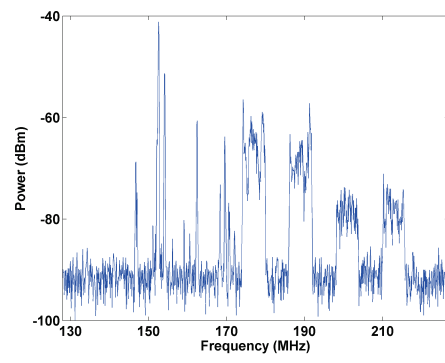

9a. Spectrum $\Psi_{274}$ that contains multiple sub-bands of RFI.

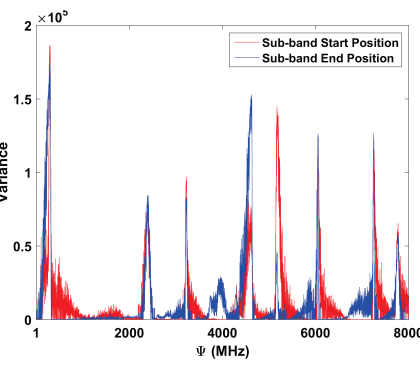

9b. Sub-band variance between the $R$ random trials.
Fig. 9. Analysis of RF spectra that result in a high $L_{1}$ distance for NSGA-II.

The computational complexity of WSMO and NSGA-II are compared in Fig. 10. As shown, the number of summations and multiplications needed for NSGA-II is significantly less than that for WSMO. NSGA-II requires approximately 6100 multiplications where WSMO requires $3 \times 10^{6}$ multiplications. NSGA-II therefore reduces the number of multiplications by a factor of 492 . The number of summations required by NSGA-II is variable and dependent on the RFI. An initial observation indicates that the number of summations is proportional to the SINR, i.e. spectra with high SINR results in more summations. The authors speculate that the high SINR indicates feasible solutions with wider sub-bands; therefore, as defined in (10), more summations are needed to process the wider sub-bands. It should be noted that the summations needed for NSGA-II is reduced $75 \%$ (as compared to WSMO).

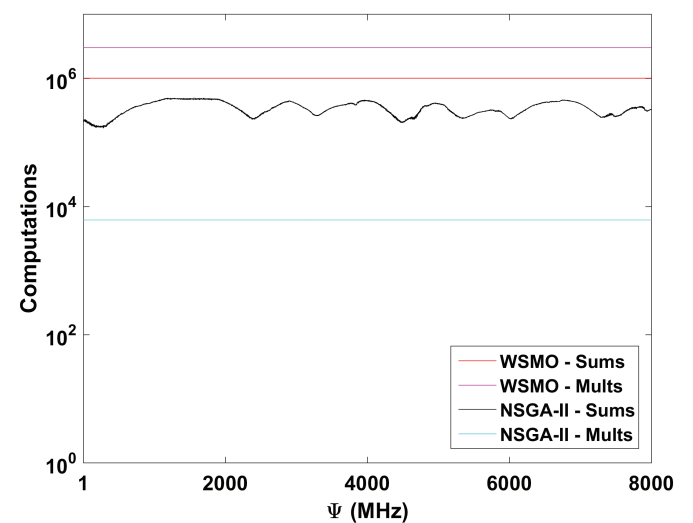

Fig. 10. Computational complexity for WSMO and NSGA-II approaches. The number of summations and multiplications needed for NSGA-II is significantly less than that for WSMO.

\section{CONCLUSION}

Two multi-objective optimization approaches were investigated for the SS-MO technique. The results indicated that utilizing the full bandwidth (without SS-MO) in the presence of RFI results in reduced SINR, with some results below the SINR boundary limit of $-10 \mathrm{~dB}$. WSMO and NSGA-II met the SINR boundary limit requirements for all processed spectra, and produced SINR significantly higher than the SINR produced using the full bandwidth. $L_{1}$ distance results indicated a significant lower error produced by WSMO and NSGA-II as compared with the full bandwidth, which further justifies the utilization of both approaches and the SS-MO technique.

It was also shown that NSGA-II results in a large $L_{l}$ distance for a small number of spectra. NSGA-II results for thermal noise indicate that the full bandwidth (the best solution available) is not consistently selected, however NSGA-II does generate solutions "close to" the full bandwidth. Other spectra that produce large $L_{l}$ distances indicate large variation between the $R$ random trials. Hence, when processing RF spectra containing multiple sub-bands of RFI: 1) NSGA-II does not consistently converge to the best-known Pareto optimal set; 2) selection of $\left\{\beta_{i}^{*}, f_{j}^{*}\right\}$ by NSGA-II is highly dependent on the random locations of the initial population.

The computational complexity of NSGA-II was significantly less than that of WSMO. NSGA-II requires approximately 6100 multiplications where WSMO requires $3 \times 10^{6}$ multiplications, a reduction of over 492 times. The number of summations required by NSGA-II is variable and dependent on the RFI; however, the summations needed for NSGA-II is less than that for WSMO.

\section{REFERENCES}

[1] Federal Communication Commission, "Auction of Advanced Wireless Services (AWS-3) Licenses Closes," Wash. D.C., DA 15-131, 2015.

[2] M. Labib, J.H. Reed, A.F. Martone, A.I. Zaghloul, "Coexistence between Radar and LTE-U Systems: Survey on the $5 \mathrm{GHz}$ Band," in Proceedings of the 2014 USNC-URSI Radio Science Meeting, Boulder, CO, Jan 2016.

[3] A.F. Martone, "Cognitive Radar Demystified," URSI Bulletin, no. 350, pp. 10-22, September 2014.

[4] T. Li, H. Yang, and Z.O. Zhou, "RFI suppression based on phase-coded stepped-frequency waveform in through-wall radar," IEEE Transactions on Geoscience and Remote Sensing, vol. 53, no. 3, pp. 1583-1591, 2015.

[5] A. Khawar, A. Abdel-Hadi, and T. C. Clancy, "Spectrum sharing between S-band radar and LTE cellular system: a spatial approach," in Proceedings of the IEEE International Symposium on Dynamic Spectrum Access Networks, pp. 7-14, McLean, Va, USA, April 2014.

[6] S.S. Bhat, R.M. Narayanan, and M. Rangaswamy, "Bandwidth sharing and scheduling formultimodal radar with communications and tracking," IETE Journal of Research, vol. 59, no. 5, pp. 551-562, 2013.

[7] J. Jakabosky, S.D. Blunt, A.F. Martone, "Incorporating hopped spectral gaps into nonrecurrent nonlinear FMCW radar emission," IEEE Intl. Workshop on Computational Advances in Multi-Sensor Adaptive Processing, Cancun, Mexico, Dec. 2015.

[8] A.F. Martone, K.D. Sherbondy, K.I. Ranney, and T.V. Dogaru, "Passive sensing for adaptable radar bandwidth," in Proceedings of the IEEE International Radar Conference, Arlington, Va, USA, May 2015.

[9] A.F. Martone, K.A. Gallagher, K.D. Sherbondy, et al., "Adaptable Bandwidth for Harmonic Step-Frequency Radar," International Journal of Antennas and Propagation, vol. 2015, July, 2015, Article ID 808093.

[10] A. Konak, D. Coitb, and A. Smith, "Multi-Objective Optimization Using Genetic Algorithms: A Tutorial," Reliability, Engineering, and System Safety, vol. 91, no. 9, pp. 992-1007, September 2006.

[11] K. Deb, A. Pratap, S. Agarwal, T. Meyarivan, "A fast and elitist multiobjective genetic algorithm: NSGA-II," IEEE Transactions on Evolutionary Computation, vol. 6, no. 2, pp. 182-197, Apr 2002.

[12] K. Deb and S. Agarwal, "Simulated binary crossover for continuous search space," Complex Systems, vol. 9, pp. 115-148, Apr 1995. 